\section{Sheet laser lightning in}

\section{Prague}

\author{
Karel Jungwirth, Jiri Ullschmied \\ Institute of Plasma Physics AS CR, Prague, Czech Republic
}

$T^{\text {n }}$ order to stop a painful brain drain and to make the domestic research career more attractive for young physicists, the Czech science policy makers promote natural centres of excellence formed around groups of the most experienced scientists or around key research facilities. In the year 2000 , several selected Czech Research Centres gained substantial additional financial support from the Ministry of Education, Youth and Sports of the Czech Republic. One of them, the Laser Plasma Research Centre in Prague, has already gained a good reputation throughout laser Europe. Its key laser facility, the Prague Asterix Laser system (PALS), lists now among the European Major Research Infrastructures. This first international multi-user high-power laser system in Europe's newly associated countries, and a European LASERNET member, celebrated recently its second anniversary.

The PALS's predecessor, the German Asterix IV high-power iodine laser, had already gained a good renown among the European laser plasma research community in the first half of the nineties of the last-century. Developed at the Max-Planck-Institut für Quantenoptik in Garching by Munich and operated there successfully since 1991, the laser suddenly faced a serious decision about its further destiny in spring 1997. Since that time it turned out that saving it for further use was very wise, and that moving the laser to Prague was really a coup for its new mother institution, the Academy of Sciences of the Czech Repüblic (AS CR). Due to its unique features, several new options and some principal innovations, the reincarnated Asterix/PALS facility is enjoying now a permanently increasing interest of both domestic and foreign researchers.

The risky operation of the laser transfer to Prague began in late summer 1997, with an ambitious goal to put the giant laser into full operation again by spring 2000 . At that time, very few people believed that the tight schedule of all the necessary operations could be maintained. For instance, in the Czech Republic no suitable housing for the laser was available. It had to be projected and built first. Nevertheless a rather small group of Czech enthusiasts managed to cope with all the obstacles that came up and fulfilled the virtually impossible. A new laser hall for the laser grew up almost overnight, the reassembling of the laser was finished and its operational tests started in late 1999, and the laser reached its full parameters in May 2000. In September 2000, the laser started to offer its beam time to European physicists.

PALS is a single-beam kilojoule-class pulsed laser. Its kilojoule output, together with a relatively long pulse $(\sim 400 \mathrm{ps})$, make it a powerful driver for various practical applications. Unlike the other, mostly solid-state, high-power lasers in Europe, PALS has an iodine gaseous medium, which gives it some unique advantages. It generates a high-quality infrared beam of an almost flat

Photo: The PALS twin target chamber. Josef Krasa (PALS ion group) adjusting diagnostics for an experiment with laser ion sources. intensity profile. A single-beam configuration of a chain of laser amplifiers permits the variation of the output beam energy over two orders of magnitude. The fundamental frequency of the beam $(1315 \mathrm{~nm})$ can be up-converted to the $2^{\text {nd }}, 3^{\text {rd }}$ and even to the ultraviolet $4^{\text {th }}$ harmonics. The laser pointing is stable over twomonths, its operation is relatively cheap. Due to all the above features, PALS is almost ideally suited for the basic and applied studies of laser interaction with matter at power density levels ranging from $10^{14}$ to $10^{16} \mathrm{~W} / \mathrm{cm}^{2}$.

Although the PALS laser still keeps the original configuration of the former Asterix, it has been upgraded by new beam lines and diagnostics options. A new original-concept twin target chamber with several beam focusing assemblies has been designed in co-operation with French laser specialists, which permits the performance of even the most sophisticated laser plasma experiments, such as development and applications of plasma soft x-ray lasers.

Current PALS main research priorities are aimed at exploiting the laser plasma as a source of soft $x$-radiation, of highly charged ions, and at relevant scientific applications. As a users facility, PALS is offering its beam time to both domestic and foreign researchers. Since September 2000 , the laser is being intensely exploited by scientists from France, Germany, Italy, Poland, Russia, Slovakia, and The Netherlands. Eighteen research projects in total, proposed both by domestic and foreign research groups, have been alieady performed at PALS during the years 20002002 , and number of new ones are planned. The research projects for PALS are peer-reviewed by an international User Selection Panel, the best projects for EU member and newly associated states being recommended for support by the European Commission's $5^{\text {th }}$ Framework Programme (Transnational Access to Major Research Infrastructures and INTAS projects). Most of the projects already supported follow two main research lines: development of ultra-bright $x$-ray sources, of the soft $x$-ray

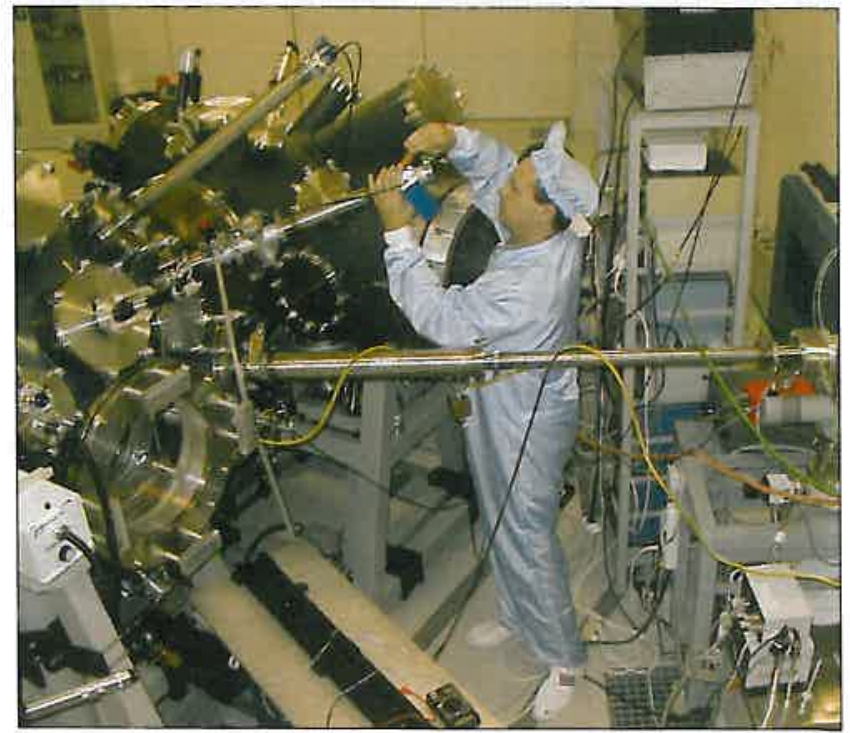


